\title{
Les échelles du transport pour compte propre, du local vers l'international : contraintes et développement
}

\author{
From local to international, the scales of own account transport: constraints and development
}

\author{
Cécilia Cruz \\ Reçu le 31 janvier 2012 ; accepté le 16 février 2013 \\ (C) IFSTTAR et Éditions NecPlus
}

\begin{abstract}
Résumé Les chargeurs adoptent des stratégies de transport en fonction de différentes contraintes notamment productives. En transport pour compte propre, la courte distance domine et donne lieu à une utilisation différente selon que l'échelle privilégiée soit locale ou régionale. Ces deux échelles prédominent chez les utilisateurs du transport pour compte propre pour des raisons liées au service. Néanmoins, quelques exemples sur une échelle nationale ou internationale subsistent et permettent de mettre en évidence la viabilité d'une organisation de transport en compte propre à ces échelles.
\end{abstract}

Mots-clés transport de marchandises · transport pour compte propre $\cdot$ stratégie territoriale $\cdot$ maillage territorial

\begin{abstract}
Transport strategies of shippers are based on constraints especially in production. In own account transport, the short distance dominates and gives rise to a different use depending on whether the scale is privileged local or regional. These latter scales predominate among users of own account transport because of service of transport. Nevertheless, a few examples of national or international organization show how own account transport is possible and viable at these scales.
\end{abstract}

Keywords freight - own account transport - spatial strategy $\cdot$ territorial grid

Ce travail de recherche a été mené dans le cadre d'une thèse au sein de l'unité de recherche SPLOTT (Systèmes productifs, logistique, organisation des transports, et travail) de l'Ifsttar et du laboratoire MRTE (Mobilités, réseaux, territoires et environnements) de l'université de Cergy-Pontoise.

Cécilia Cruz $(\square)$

Université Paris-Est, IFSTTAR, AME/DEST, F-77447

Marne-La-Vallée, France

Boulevard Newton, Champs sur Marne, F-77447

Marne-La-Vallée cedex 2

e-mail : cecilia.cruz@ifsttar.fr

\section{Introduction}

Les organisations de transport diffèrent selon les attentes des entreprises qu'elles soient industrielles ou commerciales. Ainsi pour l'acheminement de leur fret, différents choix, notamment en matière de transport routier, s'offrent aux entreprises. L'une des premières questions que les entreprises se posent en matière de transport est : qui organisera et assurera le transport des marchandises ? Certaines entreprises préférent l'assurer elles-mêmes, on parle alors de transport pour compte propre $^{1}$ tandis que d'autres confient à un prestataire la charge d'organiser et d'assurer le transport, celui-ci est alors qualifié de transport pour compte d'autrui.

Les systèmes de production ont une influence sur le transport, mais l'organisation de transport choisie a également une influence puisque des ajustements quant au nombre d'implantations et leurs localisations peuvent être nécessaires. Le transport joue un rôle dans la structure et l'organisation de l'espace comme le remarquent Rodrigue et al. [1]. Dans cet article, nous examinons l'organisation spatiale des implantations d'un type particulier d'entreprises, celles recourant au transport pour compte propre. Ainsi, suivant la répartition spatiale des implantations et les stratégies définies, l'usage et le recours au transport pour compte propre peuvent être différents.

Le transport pour compte propre est souvent présenté à travers ses courtes distances, mais à quelles organisations spatiales correspondent-elles ? Nous cherchons à montrer à l'aide de quelques exemples que cette courte distance s'inscrit dans des schémas d'organisation des implantations qui peuvent varier suivant les entreprises.

\footnotetext{
${ }^{1}$ Le transport pour compte propre a été défini en France dans la Circulaire du Ministère de l'Equipement, du Logement et des Transports $\left(\mathrm{n}^{\circ} 2000-17\right)$ relative aux titres administratifs et documents détenus par les entreprises de transport routier de marchandises et de location de véhicules industriels destiné au transport de marchandises.
} 
Les entreprises recourant au transport pour compte propre ont des stratégies et des contraintes différentes qui se reflètent dans leur usage du transport. Nous montrerons tout d'abord, à travers une analyse statistique, que même si le transport pour compte propre est utilisé principalement sur de la courte distance, il constitue une réponse à des produits et des types d'activité spécifiques. Nous nous interrogerons ensuite sur les conditions de réalisation de cette courte distance notamment en termes de besoins de proximité vis-à-vis du client. Cette courte distance peut se décliner à partir de deux types d'organisations des implantations : l'un à une échelle plus locale qui s'opère à l'aide d'un maillage territorial, l'autre à l'échelle régionale.

Enfin, nous terminerons sur un cas plus rare, l'organisation de transport en compte propre présentant une dimension nationale et/ou internationale et nous tenterons d'en comprendre les usages.

\section{Quelques caractéristiques des organisations de transport pour compte propre}

Pour mieux comprendre les singularités des organisations spatiales des utilisateurs du transport pour compte propre, il est nécessaire en premier lieu, d'exposer quelques caractéristiques importantes de ce type de transport.

\section{Des produits spécifiques... mais également des activités spécifiques}

Il est admis que le transport pour compte propre est davantage utilisé pour le transport de certains types de marchandises [2]. Les données issues de l'enquête TRM ${ }^{2}$ [3] nous permettent de constater que le transport pour compte propre est fortement utilisé pour le transport de matériaux de construction (27\% des tonnes.kilométres en 2008) ainsi que pour les minerais et déchets métallurgiques ( $31 \%$ des t.km en 2008) (Figure 1). Ainsi, la part du transport pour compte propre est la plus élevée pour les produits de plus faible valeur à la tonne comme cela a également été constaté au Royaume-Uni par Croucher [4].

Au-delà de cette distinction par produits, il convient de s'intéresser aux types d'entreprises qui ont recours au transport pour compte propre, car par exemple, la catégorie de produits manufacturés recouvre de nombreuses entreprises aux demandes de transport très diverses. L'organisation du transport de marchandises est liée aux activités de production et de commerce, Rodrigue [5]

\footnotetext{
${ }^{2}$ L'enquête Transport routier de marchandises effectuée par le ministère en charge des transports porte sur les véhicules de plus de 3,5 tonnes et de moins de 15 ans d'âge.
}

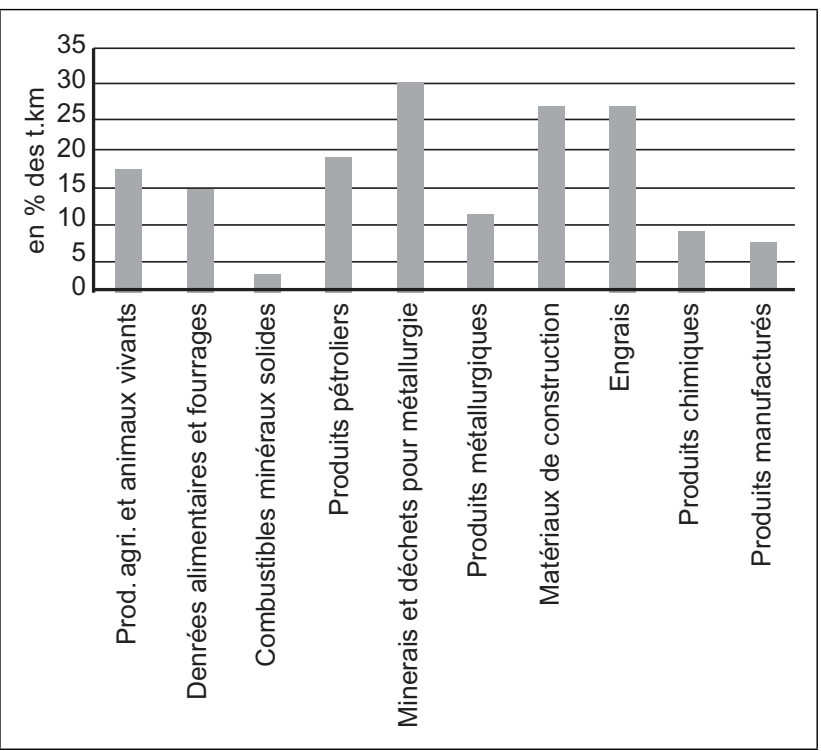

Fig. 1 Part du transport pour compte propre par type de produits en France en 2008 (en tonnes.kilomètres)

Source : données Enquêtes TRM, SOeS, MEDDTL

le qualifie d'ailleurs de demande dérivée, la vision du transport pour compte propre à travers les types de produits doit être complétée. Les seules caractéristiques du produit n'expliquent pas un choix de transport, il est important d'examiner les contraintes productives des entreprises. Les enquêtes chargeurs menées par l'Inrets (Institut national de recherche sur les transports et leur sécurité) en 1988 (Chargeurs 88) et 2004 (Echo - Envois chargeurs opérateurs de transport) permettent de mieux appréhender ce lien entre les activités économiques et le transport qui en résulte.

Ainsi, si le transport pour compte propre est davantage utilisé pour certains types de produits, une approche par les activités s'avère plus discriminante. Les enquêtes chargeurs sont, à cet égard, utiles car elles permettent d'effectuer une différenciation selon le type d'activité, commercial ou industriel ${ }^{3}$. Depuis 1988, l'importance des commerces de gros dans le transport pour compte propre a tendance à se renforcer. En effet, comme le montre la Figure 2, la diminution a été plus forte dans les industries et plus particulièrement dans l'industrie agroalimentaire : la part du compte propre est passée de $61 \%$ en 1988 à $28 \%$ en 2004.

\footnotetext{
${ }^{3}$ Les champs couverts par l'enquête sont l'ensemble des établissements de 10 salariés et plus, appartenant aux secteurs de l'industrie (hors industries extractives) et des commerces de gros. Le champ de l'enquête Echo s'est élargi depuis la précédente enquête en 1988 : les coopératives agricoles, la vente par correspondance, les entrepôts et les centres de traitement des déchets industriels. Pour ces secteurs nouvellement enquêtés, le seuil de 6 salariés a été retenu. Seuls, les expéditeurs d'envois d'au moins $1 \mathrm{~kg}$ ont été sélectionnés. Pour plus d'informations sur la méthodologie de l'enquête ainsi que ses principaux résultats, voir Guilbault et al. (2008) [6].
} 


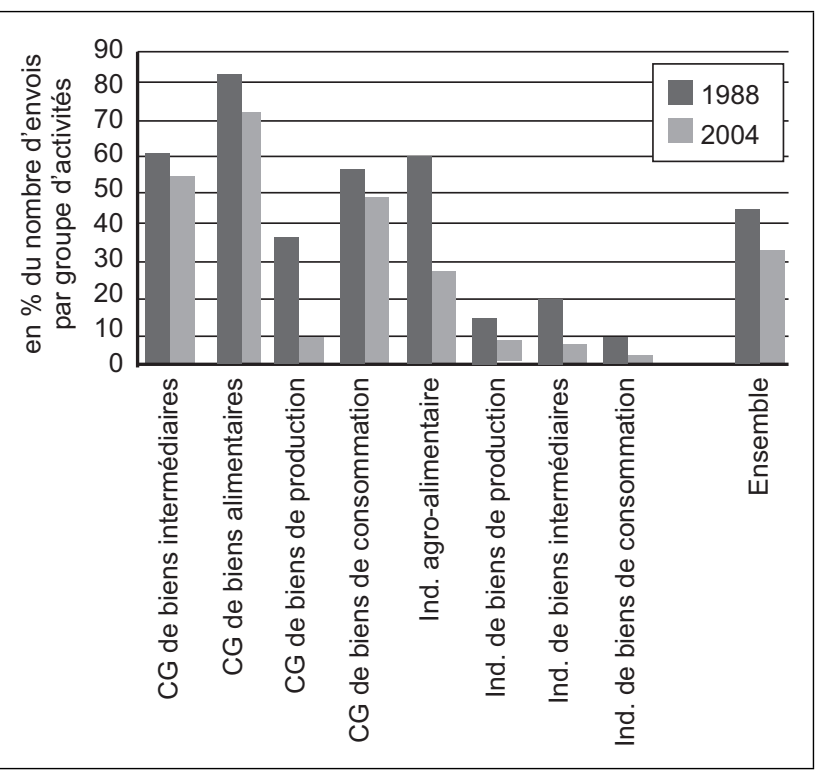

Fig. 2 Part du transport pour compte propre en nombre d'envois par groupe d'activité en 1988 et 2004

Source : données Enquête Chargeurs, 1988, INRETS ; Enquête ECHO (champ 1988), 2004, INRETS - Calculs de l'auteur.

Cette distinction commerce de gros/industries permet de mettre en évidence quels sont les types d'utilisateurs du transport pour compte propre. Dans le commerce de gros, le transport est perçu comme une activité stratégique puisque ces entreprises ne fabriquent pas de produits et ne possèdent donc que peu ou pas de produits différents de leurs concurrents. La différenciation s'effectue par le service rendu au client et le transport constitue un élément clé de cette stratégie.

Ainsi, les chargeurs utilisant le transport pour compte propre répondent à une demande spécifique avec des moyens propres.

\section{Persistance des courtes distances dans le transport pour compte propre}

Depuis la fin des années 1980, la part du transport pour compte propre dans le transport routier diminue même si depuis la crise de 2008, elle augmente sensiblement (19\% des t.km du transport routier en 2011). Cette faible part dans le transport routier n'est pas sans lien avec l'une des caractéristiques principales du compte propre : sa courte distance. Celle-ci a été constatée dans différentes études de la CEMT [7], Patier [8], Savy [3].

Le transport pour compte propre est majoritairement présent sur les courtes distances depuis plusieurs décennies et la tendance se poursuit. En effet, la part des distances de plus de $150 \mathrm{~km}$ est passée en France de $50 \%$ des t.km en compte propre en 1996 à seulement $40 \%$ des t.km en 2011 (Tableau 1).
Tableau 1 Le transport routier de marchandises en France ${ }^{4}$ en fonction du type de régime en 1996 et en 2011 (en tonnes.kilomètres)

\begin{tabular}{llllll}
\hline & $\mathbf{1 9 9 6}$ & & & $\mathbf{2 0 1 1}$ & \\
\cline { 2 - 3 } \cline { 5 - 6 } & $\mathbf{C P}$ & $\mathbf{C A}$ & & $\mathbf{C P}$ & $\mathbf{C A}$ \\
\hline Moins de $150 \mathrm{~km}$ & 18,5 & 23,6 & & 19,6 & 35,2 \\
$150 \mathrm{~km}$ et plus & 18,3 & 98,8 & & 13,1 & 110,1 \\
Total & 36,8 & 122,4 & & 32,7 & 145,3 \\
\hline CP : compte propre, CA : compte d'autrui. & & & \\
Source : Données Enquêtes TRM, SOeS, MEDDTL & & \\
\hline
\end{tabular}

\section{Le besoin de proximité avec le client au cour des organisations de transport pour compte propre}

La courte distance est un élément caractéristique du compte propre et celle-ci est liée au besoin de proximité. En effet, dans les activités recourant au compte propre, le lien avec le client s'avère être un élément essentiel de leur activité.

Les liens entre un chargeur et son destinataire sont plus forts en cas de recours au transport pour compte propre comme le montre la fréquence de leurs échanges. Des résultats obtenus à partir de l'enquête Echo illustrent cet écart : pour un même couple origine-destination, 524 envois par an sont effectués, en moyenne, dans le cas d'une utilisation du transport pour compte propre contre 147 dans le cas d'un recours au transport pour compte d'autrui. Ces échanges plus fréquents (plusieurs envois par jour) témoignent d'une relation régulière entre les deux parties et de fait, nécessitent une proximité géographique avec le client. Cette forte fréquence s'explique par la forte proportion de commerces de gros parmi les utilisateurs du transport pour compte propre $(62 \%$ des envois en compte propre) bien qu'ils ne représentent que $26 \%$ de l'échantillon des établissements enquêtés 5 .

Ces relations régulières et fréquentes induisent donc, de la part de l'entreprise, une bonne connaissance de ses clients. En effet, dans les commerces de gros, ces derniers ont une mission de conseil envers leurs clients. Ce lien peut être formalisé dans certains secteurs d'activité tels que le commerce de gros de boissons, par des contrats d'exclusivité d'une durée de cinq ans, durée maximum en raison d'un règlement européen.

Cette connaissance du client et la fréquence des contacts soulignent le caractère routinier des transports en compte

\footnotetext{
${ }^{4}$ Transport national et partie française de l'international.

${ }^{5}$ Données Enquête Echo, 2004, Inrets - Calculs de l'auteur.
} 
propre. En effet, plus de la moitié des envois en compte propre $(55 \% \text { des envois })^{6}$ sont effectués dans le cadre d'un programme prétabli tandis qu'en compte d'autrui, la proportion s'élève à un peu plus d'un tiers (37\% des envois). Si le caractère routinier est présent, deux éléments sont au cœur de ces organisations et peuvent expliquer ce besoin de proximité : la flexibilité et la réactivité. Les délais de livraison sont plus courts chez les utilisateurs du compte propre lorsque l'envoi n'est pas programmé, notamment en milieu urbain où le transport pour compte propre est très présent [9]. Ainsi, le transport pour compte propre permet de répondre rapidement à une demande urgente puisqu'il n'est pas nécessaire de faire appel à un transporteur. De plus, étant donné la taille des envois, une consolidation serait nécessaire dans le transport pour compte d'autrui. En outre, les délais de livraison étant courts, les horaires d'enlèvement et de livraison dans le compte d'autrui ne sont pas adaptés. De précédents travaux ont mis en exergue cette inadéquation des horaires du transport pour compte d'autrui en milieu urbain [9].

Ce besoin de proximité va de pair avec une courte distance, mais comment se construit-elle ? Elle se décline sous différentes formes d'organisation spatiale qui sont à l'origine de cette courte distance : le maillage territorial et une organisation plus régionale.

\section{Une courte distance, résultat d'un maillage territorial}

Les courtes distances parcourues dans le cadre d'un transport pour compte propre résultent, dans certains cas, d'une stratégie de maillage territorial. Qu'entend-on par maillage territorial ? D'après Brunet, le maillage « tradui[ $\mathrm{t}]$ des effets spatiaux particuliers et des dynamiques de l'espace, où l'on retrouve comme il se doit, les lois de la distance et de l'espacement »[10]. Il s'agit donc de découper l'espace en mailles. Nous entendons par maillage territorial, la répartition spatiale des établissements ${ }^{7} \mathrm{~d}^{\prime}$ une même entreprise qui permet de découper l'espace de manière plus fine. ${ }^{8}$ Cette organisation spatiale en maillage territorial permet à l'entreprise de conserver des courtes distances, voire même dans certains cas de très courtes distances.

Dans les organisations spatiales de type maillage, le choix $\mathrm{du}$ transport pour compte propre s'effectue pour des raisons de proximité. L'entreprise est présente sur l'ensemble de la

\footnotetext{
${ }^{6}$ Source : Données Enquête Echo, 2004, Inrets - Calculs de l'auteur.

${ }^{7}$ L'établissement est une unité de production géographiquement individualisée, mais juridiquement dépendante de l'entreprise. Source : Site Internet Insee : www.insee.fr

${ }^{8}$ Pour une discussion sur les termes de maillage et treillage, voir [11].
}

France par le biais de plusieurs établissements répartis sur l'ensemble du territoire et cherche à augmenter le nombre de ses installations afin de maintenir et développer une certaine proximité avec ses clients.

Ce maillage territorial s'effectue pour deux raisons principales, soit en raison de contraintes liées au produit, soit pour des raisons liées à une stratégie marketing.

\section{Un maillage territorial, résultat de contraintes}

Certaines activités, pour des raisons liées à la spécificité $\mathrm{du}$ produit, maillent l'espace, c'est notamment le cas du gaz. L'activité de l'entreprise distributrice de gaz, dont nous avons rencontré l'un des responsables transport ${ }^{9}$, se décompose en deux segments de marché : d'une part, les bouteilles en gaz dont les utilisateurs sont des particuliers et d'autre part, le marché vrac livré en citerne (Figure 3) dont les clients sont indifféremment des particuliers ou des professionnels.

Pour le vrac, l'entreprise utilise une cinquantaine de points de rechargement qui permettent de recharger les camions pour redistribuer vers des clients dits « vrac» (Figure 3). L'entreprise possède 17 relais-vrac dont les capacités varient entre $50 \mathrm{t}$ et $500 \mathrm{t}$, et utilise d'autres points de rechargement qui appartiennent à des concurrents.

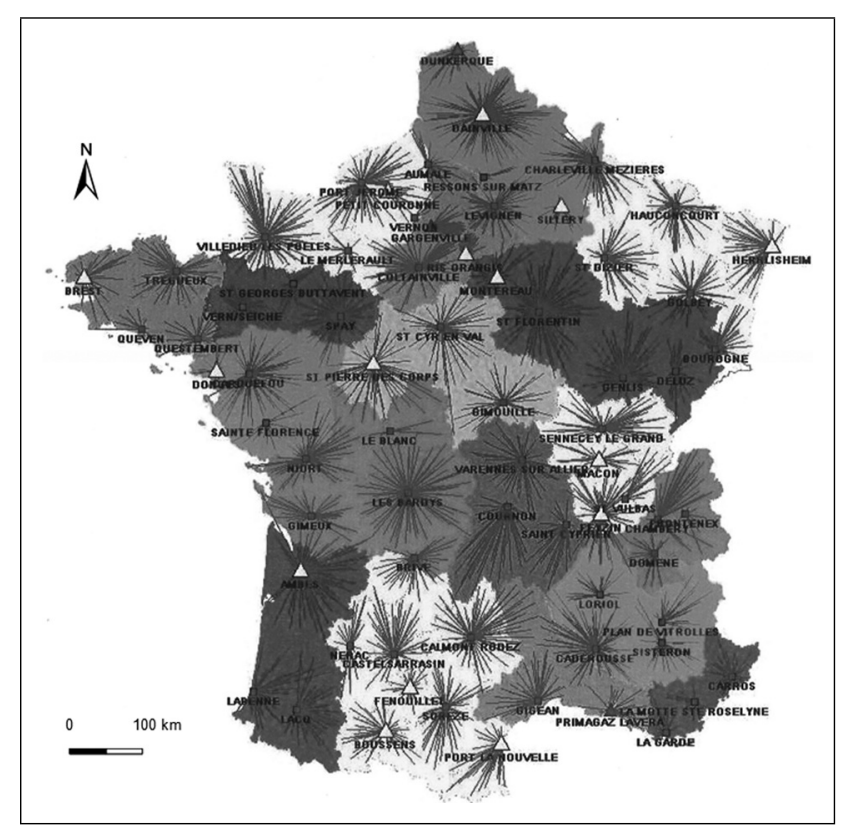

Fig. 3 Plan de desserte prévue de l'activité vrac de gaz d'une entreprise distributrice en 2007

Source : document entreprise.

\footnotetext{
${ }^{9}$ Entretien le 11 avril 2008.
} 
Ces points de rechargement «partagés » fonctionnent dans un système d'échange comme dans le pétrole. L'entreprise achète du gaz à ses concurrents sur ces points de rechargement afin de limiter les distances parcourues à partir de ses propres installations et ainsi les coûts de transport. Le partage de celles-ci permet de mieux mailler le territoire, et ainsi limiter le nombre des installations industrielles.

Le transport pour compte d'autrui est utilisé pour l'approvisionnement des relais-vracs tandis que le transport pour compte propre est utilisé dans le cadre des livraisons aux clients. Le contact avec le client est primordial pour cette entreprise. Ainsi, lors de la livraison le chauffeur peut transmettre des informations aux clients par le biais de brochures. Cette prestation est difficilement réalisable dans le cadre d'un transport pour compte d'autrui.

Pour l'organisation des trafics entre les sites partagés et les sites de l'entreprise, les responsables transport cherchent d'abord, à effectuer un maximum de trafics au sein de l'entreprise, puis lorsqu'ils ont recours aux sites des concurrents, c'est le nombre inférieur de kilomètres qui permettra de réaliser des économies sur le coût total. Un seuil de stockage élevé induit l'existence d'un danger potentiel, celui-ci implique un classement en Installation contrôlée pour la protection de l'environnement (ICPE), les installations représentant des risques majeurs sont également soumises à la réglementation européenne Seveso II. Suite à la directive européenne $\mathrm{n}^{\circ}$ 96/82 de $1996^{10}$, dite Directive Seveso II, deux catégories d'établissements sont distinguées : les établissements à haut risque (seuil haut) correspondant souvent aux installations classées soumises à autorisation avec servitudes et les établissements à risque modéré (seuil bas) correspondant souvent aux installations soumises à autorisation mais dont le potentiel de danger est considéré comme important. Selon des informations transmises en 2010, par le Chef du service des risques technologiques au ministère en charge des Transports, pour le stockage de GPL, les seuils sont les suivants :

- installation dites « seuil haut » : 200 tonnes ;

- installation dites « seuil bas » : 50 tonnes.

Le stockage est réglementé, ceci s'avère fondamental en termes logistiques, puisque les capacités ne peuvent pas être dépassées pour des raisons de sécurité.

Les prévisions sont très importantes dans la gestion des stocks. En cas de mauvaise prévision, si sa capacité de stockage est dépassée avec le contenu de la livraison, il n'est pas possible d'approvisionner le dépôt. Cette activité est très particulière dans la mesure où la taille du stockage conditionne la zone rattachée. En effet, en raison des risques

\footnotetext{
${ }^{10}$ Directive $\mathrm{n}^{\circ}$ 96/82 concernant la maîtrise des dangers liés aux accidents majeurs impliquant des substances dangereuses.
}

liés au stockage de produits considérés comme dangereux, la capacité de stockage à proximité d'agglomérations est limitée, d'où une utilisation partagée des sites. Le maillage n'est cependant pas aussi dense que dans le cas du commerce de gros en matériaux de construction, exemple que nous développons ci-après. Le nombre de plates-formes est plus réduit en raison de contraintes de sécurité. Pour chaque nouvelle plate-forme, une autorisation doit être délivrée par les autorités en raison des risques.

D'une année sur l'autre, les limites des zones sont variables. Ainsi, si un concurrent décide d'augmenter les prix sur les points de rechargement, l'organisation de transport doit être revue afin de rechercher une solution plus pertinente économiquement. L'entreprise a recours à un système Advanced Planning Scheduling (APS) qui permet de prendre en compte un grand nombre de paramètres tels que la distance à parcourir, les frais de stockage et de chargement, ou encore le coût de l'approvisionnement du site et le prix du gaz. Cet outil utilise des prévisions de vente par client agrégées par canton sur l'ensemble de l'année. L'APS établit un schéma idéal qu'il faut ensuite modifier dans l'année en fonction de la demande, il constitue toutefois une base de travail utile dans la gestion des flux. L'utilisation de ce système se fait de manière progressive depuis cinq ans ${ }^{11}$; ainsi au départ, seuls les coûts de la distribution locale ont été pris en compte. Cet outil, qui permet la modulation des zones, entraîne des changements dans le système de répartition des achats à des concurrents en cas d'augmentation de ses tarifs. Par exemple, pour des achats de $15000 \mathrm{t}$ auparavant, il peut être décidé de passer à 2000 t. D'un point de vue économique, il vaut mieux parcourir plus de kilomètres, car cela reviendra moins cher que les $10 €$ à $12 €$ demandés par leurs concurrents pour l'utilisation de leurs stocks ${ }^{12}$.

Les contraintes liées aux produits peuvent entraîner de nouvelles implantations comme l'illustre le cas d'un commerce de gros de matériaux de construction, d'appareils sanitaires et de bois et produits dérivés qui a recours au compte propre pour ses livraisons. L'activité de cette entreprise est liée aux concentrations de population, d'où la forte proportion des agences en particulier en région parisienne, la vallée du Rhône et le long de la côte méditerranéenne (Figure 4). Une forte activité permet d'implanter de nouvelles installations qui maillent le territoire et ainsi de limiter les distances. La proximité spatiale entre le client et le chargeur est essentielle comme nous l'avons évoqué précédemment. Il existe une volonté de développer une relation de proximité. Ces nouvelles installations permettent de diminuer les distances parcourues et donc, les coûts de

\footnotetext{
${ }^{11}$ Entretien avec le directeur de l'Agence commerciale, le 11 avril 2008.

12 Ibid.
} 


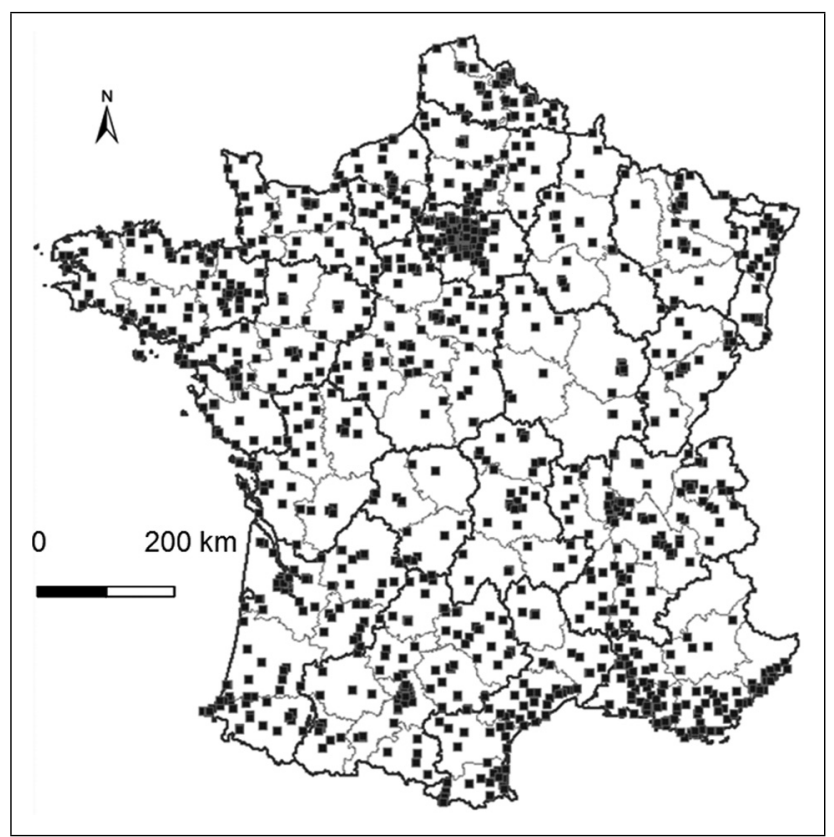

Fig. 4 Les agences d'un commerce de gros en matériaux de construction, d'appareils sanitaires et de bois et produits dérivés en 2007

Source : document entreprise.

transport qui sont élevés pour ce type de produit en proportion de leur prix de vente. Le maillage territorial résulte d'un équilibre entre le coût de transport et le coût de gestion d'une nouvelle implantation; il se traduit par la recherche de l'optimum entre concentration et dispersion spatiale.

Comme pour les matériaux de construction, les produits de nutrition animale présentent un prix de vente à la tonne faible. D'après un chargé de mission Économie et environnement au Syndicat national de l'industrie et la nutrition animale (Snia), $80 \%$ du prix de vente de l'aliment est constitué par la matière première ${ }^{13}$. En conséquence, le taux de rentabilité est de l'ordre de $0,5 \%$ à $1 \%$ en moyenne ${ }^{14}$, ceci démontre que c'est une industrie de volume.

Le choix du transport pour compte propre est en partie lié à un manque d'offre sur un matériel spécifique : les citernes spécialisées pour aliments qui sont compartimentées. Ces véhicules sont modulables suivant les commandes et répondent bien aux variabilités des demandes des agriculteurs. Différents types de produits peuvent être transportés en même temps afin d'optimiser les tournées.

Les trois cent quarante usines sont réparties sur tout le territoire afin de minimiser les distances de déplacements (Figure 4). Les coûts de transport étant élevés, il est plus rentable, d'après notre interlocuteur, de construire une usine

\footnotetext{
${ }^{13}$ Entretien le 9 juillet 2009.

${ }^{14}$ Source : ibid.
}

lorsque la distance dépasse $150 \mathrm{~km}$ à $200 \mathrm{~km}$. Au-delà de cette limite, il est difficile d'être concurrentiel d'autant que le transport n'est pas facturé en tant que tel. L'ensemble des agriculteurs achètent les produits au même prix, il n'y a pas de modulation en fonction de la distance de l'agriculteur à l'usine puisqu'il n'existe pas de frais de livraison. Ceci explique la permanence de flux locaux et la volonté de les maintenir.

La Figure 5 met en évidence une plus forte concentration des industries dans la région Bretagne. Cette région regroupe $23 \%$ de l'effectif salarié de l'industrie de fabrication d'aliments pour animaux ${ }^{15}$. Les élevages sont majoritairement présents dans cette région. Ils représentent $10 \%$ du nombre de bovins, $56 \%$ du nombre de porcins et $36 \%$ des poules et poulets élevés en France métropolitaine ${ }^{16}$. Ceci explique la plus forte concentration des industries de nutrition animale dans cette région.

Dans les différents exemples exposés, nous constatons que la part du coût de transport dans le prix de vente de ces produits est élevée. En effet, ces produits pondéreux présentent une faible valeur à la tonne. Ce coût élevé du transport à la tonne est un frein à l'augmentation de la distance parcourue. Aussi, les différentes entreprises multiplient leurs implantations pour tenter de maîtriser la part du coût de transport dans le prix de vente du produit, mais la réactivité et la souplesse vis-à-vis du client sont des éléments déterminants pour comprendre cette stratégie de maillage des implantations ainsi que le recours au transport pour compte propre.

\section{Un maillage territorial, résultat d'une stratégie marketing}

La courte distance ne s'explique pas uniquement par les contraintes des produits mais également par le niveau de service défini par l'entreprise. Ainsi, le maillage territorial peut également être le résultat d'une stratégie marketing comme c'est le cas d'un commerce de gros de produits d'épicerie. Cette entreprise cherche à étendre son aire de chalandise par le biais d'un maillage territorial.

La distance en temps et le nombre de livraisons dans un espace donné sont des éléments déterminants dans la définition des différentes zones de livraison. Ainsi, lorsqu'une zone est à une heure et demi d'une plate-forme et où le volume à livrer correspond à trois camions, l'entreprise crée une antenne avec une navette pour être à nouveau dans une logique de distribution de proximité. En maintenant

\footnotetext{
${ }^{15}$ Données Enquête annuelle d'entreprise (20 salariés et plus), 2008, Agreste - Calculs de l'auteur.

${ }^{16}$ Données Statistique agricole annuelle, Agreste, 2008 - Calculs de l'auteur.
} 


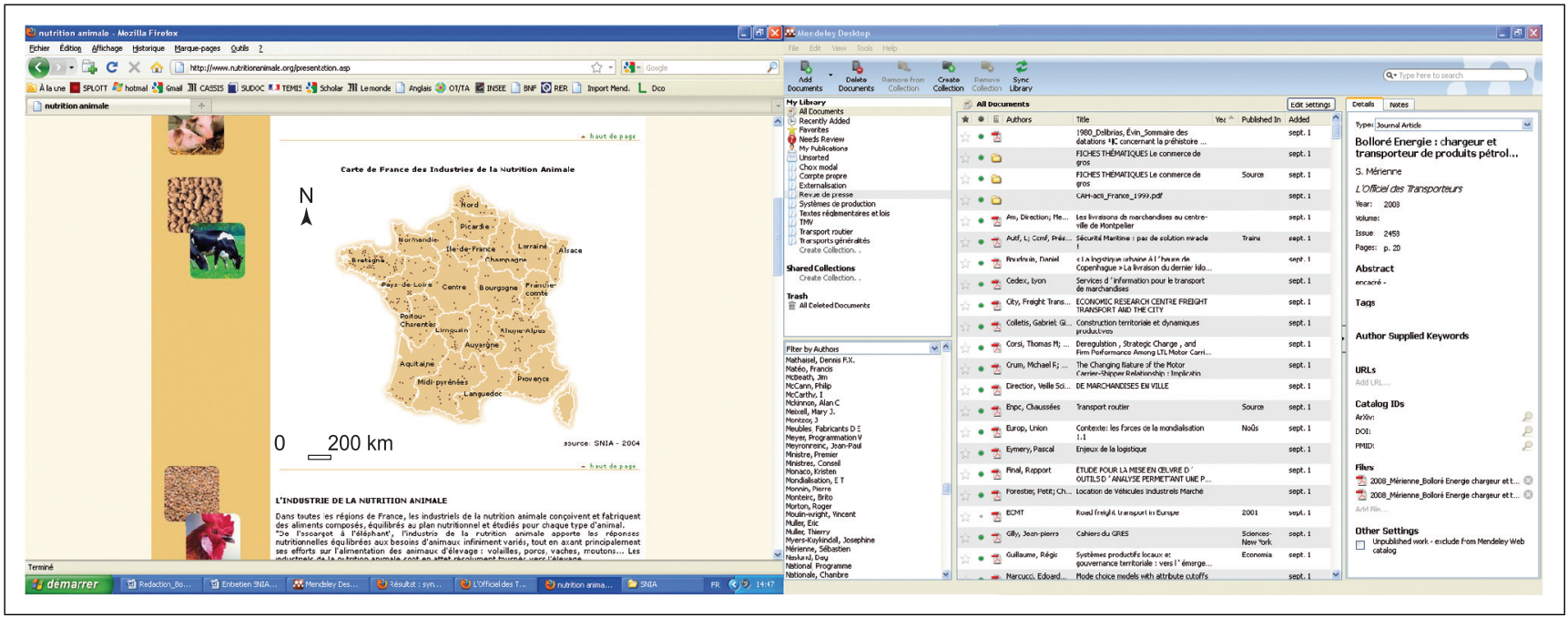

Fig. 5 Les industries de nutrition animale en 2004

Source : d'après le site internet SNIA : http://www.nutritionanimale.org (le 11 octobre 2010).

une distribution de proximité, l'entreprise souhaite limiter l'entrée de concurrents sur ces territoires. La distribution en compte propre fonctionne comme un verrou vis-à-vis de la concurrence. L'optimisation du véhicule par un transporteur, pour les zones jugées les moins pertinentes pour le développement de l'entreprise, peut permettre à des concurrents de profiter de la mutualisation sur ces territoires. Pour y remédier, cette entreprise a donc une logique de saturation y compris auprès des transporteurs chargés des approvisionnements. La maîtrise du transport constitue un enjeu dans l'activité de cette entreprise jusque dans ses approvisionnements.

Ce maillage territorial des implantations peut, en partie, expliquer le renforcement de la courte distance dans le transport pour compte propre. Cette organisation vise à minimiser les distances parcourues par le biais d'un maillage des implantations qui se densifie au fil du temps avec les nouveaux clients. La concentration des implantations autour des grands pôles urbains permet de maintenir une fiabilité des délais élevée et des délais de livraison courts ce qui est important pour des produits tels que les matériaux de construction. Le cas du gaz apparaît comme particulier puisque très réglementé en raison de sa dangerosité. Pourtant, même si les installations sont contraintes par des autorisations spéciales, l'entreprise distributrice de gaz est également dans cette stratégie de densifier son réseau et elle cherche à être au plus près de ses clients pour répondre plus rapidement aux demandes qui peuvent varier fortement d'un jour sur l'autre.

Ces entreprises sont présentes dans l'ensemble de la France mais avec une volonté de conserver une proximité géographique avec le client afin d'avoir une meilleure connaissance des besoins des différentes zones de chalandise. Le transport en compte propre s'inscrit en continuité avec la volonté de proximité avec le client, donc de réponse rapide à ses demandes de livraison. C'est notamment le cas dans les commerces de gros où les livraisons effectuées à $\mathrm{J}+1$ sont fréquentes.

\section{Les organisations à vocation régionale : des courtes distances mais une organisation différente}

Nous avons identifié un deuxième type d'organisation de transport en compte propre à une échelle plus régionale. Celui-ci se caractérise par une action centrée sur un territoire de portée régionale et non pas à partir d'une multitude de points de départ comme dans le précédent type, mais à partir d'une base régionale. Dans ce cas, la courte distance est encore présente, mais de façon différente.

Nous pouvons distinguer deux sous-types en fonction de la taille de l'entreprise et du nombre d'établissements. Le premier est le fait d'entreprises mono-établissement de petite ou moyenne taille tandis que le second révèle une forte concentration des flux sur une région qui justifie à elle seule une organisation en compte propre.

Le premier sous-type regroupe des petites et moyennes entreprises qui ne desservent qu'une ou deux régions en compte propre. Ces entreprises sont souvent monoétablissement. Elles ne desservent qu'une ou deux régions en compte propre. Ceci s'explique par leurs moyens limités tant humains que techniques. Si leur activité s'étend sur un territoire plus vaste, elles ne conservent en propre qu'une seule région qui peut coïncider avec une région 
administrative mais ce n'est pas toujours le cas. Ainsi, par exemple, dans un commerce de gros de viande ${ }^{17}$ situé dans le Berry, la zone desservie en propre s'étend à $150 \mathrm{~km}$ autour de sa base (soit $150 \mathrm{~km}$ * aller et retour $=300 \mathrm{~km}$ ) correspondant à la distance maximum parcourue par ses chauffeurs en une journée.

Comme le constatent Chanut et al. [12], « les entreprises de commerce de gros sont souvent de petites structures avec des zones de chalandise assez limitées; leur avantage compétitif s'analyse en termes de proximité avec la clientèle et de connaissance du terrain ». Comme dans le cas du maillage territorial, il existe une relation de proximité avec le client, toutefois, elle n'est pas vouée à s'étendre sur d'autres régions en raison d'une forte prépondérance du marché local et ici, les implantations ne sont pas multiples. Les entreprises de ce type s'inscrivent dans un fort ancrage régional. De fait, ce qui distingue ces entreprises des autres c'est la connaissance du client et de ses attentes, ainsi dans le cas d'un commerce de gros de viande dont nous avons rencontré le directeur transport, le chauffeur possède dans certains cas les clés de la boucherie afin d'y livrer la marchandise en l'absence du client. Cette relation de confiance se construit au fur et à mesure du temps. Le transport pour compte d'autrui ne présente pas d'intérêt dans ce cas, car il est impossible pour le chargeur de certifier que c'est toujours le même chauffeur qui livre alors qu'il n'a aucun pouvoir de décision. Il est alors difficile d'établir une relation de confiance avec le boucher dans le cas d'un transport pour compte d'autrui, d'où l'importance pour cette entreprise de maintenir une activité de transport avec des moyens propres.

Ce qui caractérise le second sous-type ce sont des entreprises de plus de 250 salariés qui possèdent des moyens humains importants et peuvent avoir plusieurs établissements dans différentes régions. Dans ce cas, l'activité de l'entreprise s'étend bien souvent sur l'ensemble de la France, mais l'activité est découpée selon ses implantations. Il existe une forte concentration sur une ou deux régions, justifiant de ce fait l'intérêt d'une desserte en compte propre uniquement sur ces territoires.

L'utilisation du transport pour compte propre dans ces entreprises correspond aux lieux où l'activité est la plus fréquente et la plus forte en termes de tonnage transporté. Le choix entre compte propre et compte d'autrui s'effectue selon des critères de fréquence et de tonnage émis, il s'agit d'effectuer un choix en fonction de critères économiques pour les distances les plus éloignées [9].

L'organisation régionale se distingue principalement de l'organisation en maillage territorial par une absence de

\footnotetext{
${ }^{17}$ L'entreprise emploie 40 personnes. Source : entretien avec le responsable transport, le 4 avril 2008.
}

volonté de l'entreprise de multiplier les implantations pour offrir davantage de service et de proximité aux clients puisque les clients situés hors de la zone compte propre de départ sont livrés en compte d'autrui. Cette stratégie plus régionale s'organise à partir d'un point central pour la desserte et n'a pas vocation à s'étendre. Tout comme dans les organisations de maillage territorial, la courte distance est une caractéristique importante. Les questions temporelles sont également centrales dans ces organisations de portée plus régionale puisque si la destination est trop éloignée, les envois seront confiés à un prestataire de transport. L'objectif pour ces entreprises est de développer en priorité l'activité autour des implantations afin d'entretenir cette proximité avec les clients.

\section{La longue distance, un cas rare parmi les organisations de transport en compte propre}

L'activité de transport à plus longue distance reste marginale dans l'ensemble de l'activité de compte propre comme en témoigne la très faible présence sur les distances supérieures à $500 \mathrm{~km}$ : d'après des résultats issus de l'enquête Echo, les envois en compte propre représentent moins de $1 \%$ des envois routiers à plus de $500 \mathrm{~km}$. Néanmoins quelques cas d'entreprise subsistent dans le temps. Comment s'organisent-elles sur ces longues distances qui ne sont pas a priori le domaine privilégié des chargeurs recourant au compte propre?

Lors de nos recherches, nous avons rencontré deux entreprises. Bien qu'elles ne soient pas représentatives au sens strict du terme, ces deux cas nous permettent de mieux appréhender le transport pour compte propre sur longue distance et de comprendre ce qui incite ces entreprises à maintenir une desserte en compte propre sur longue distance.

Le premier exemple est un traiteur localisé en région parisienne qui ne déplace pas que des marchandises, mais également des moyens humains. En effet, les prestations fournies comprennent généralement du personnel pour assurer le bon déroulement des cocktails ou repas. Ainsi, ce ne sont pas uniquement des marchandises qui sont transportées mais également un service et le savoir-faire des serveurs. Cette entreprise se caractérise par son segment d'activité haut de gamme. La portée nationale de son transport pour compte propre s'explique par une volonté de conserver une image de marque qui est très importante dans le luxe. Toutefois, les transports nationaux restent marginaux dans son activité, car celle-ci se concentre avant tout en région parisienne. Les interventions à l'international correspondent à des événements exceptionnels et ne font pas nécessairement l'objet d'un transport en camion, mais 
plutôt en avion en raison de la périssabilité des produits transportés.

Le second exemple est différent puisque toute l'activité s'organise autour d'un pôle de production ${ }^{18}$ situé dans la région Rhône-Alpes. Il s'agit d'une société familiale fabriquant des meubles. Le transport réalisé par des moyens propres est une composante essentielle de son schéma de production. Le transport est imbriqué à la chaîne de production. Ainsi, afin d'assurer la livraison par des moyens propres, l'entreprise s'interroge en amont sur ses capacités de transport, ce qui est important compte tenu de l'étendue de la zone de livraison en propre. La date de livraison est établie à partir de la date de commande, elle varie entre 21 et 28 jours en fonction des temps de séchage plus ou moins longs selon les produits. Cette entreprise ne possède pas de stock et fonctionne uniquement à la commande.

Les clients sont livrés par des moyens propres de l'entreprise dans l'ensemble de la France à l'exception de la Bretagne, mais également en Europe jusqu'en Norvège et en Suède. L'exclusion de la Bretagne s'explique par des volumes insuffisants pour présenter un intérêt économique pour le fabricant, mais également par la présence de prestataires spécialisés et compétents sur ce segment de livraison. À la différence de la Bretagne, la livraison jusqu'en Suède résulte davantage de contraintes imposées que d'un réel choix. Le responsable transport de cette entreprise ${ }^{19}$ mentionne d'ailleurs l'absence de réelle alternative pour la livraison de ses produits. En effet, il existe peu de transporteurs spécialisés sur ce segment ${ }^{20}$ et aucun pour l'Europe du Nord. Les chauffeurs partent alors pour 15 jours ce qui représente un coût de main-d'œuvre élevé. Cette livraison en compte propre s'explique par la spécificité des produits. Les meubles montés sont fragiles et la palettisation doit être évitée. Les chauffeurs reçoivent d'ailleurs une formation au début de leur recrutement pour le chargement et le déchargement des marchandises. De plus, lors des premières livraisons, ils sont accompagnés d'un chauffeur plus expérimenté afin de limiter les dommages causés aux meubles.

\footnotetext{
${ }^{18}$ Nous parlons ici de pôle car l'entreprise détient plusieurs usines et lieux de stockage distants les uns des autres de quelques kilomètres.

${ }^{19}$ Entretien avec le directeur Transport et le logisticien Transport, le 23 juin 2008.

20 Ce marché était qualifié de marché de niche en 2004 (L'Officiel des transporteurs magazine, suppl. au $\mathrm{n}^{\circ} 2287$ de L'Officiel des transporteurs). L'Officiel des transporteurs répertorie, pour l'année 2009, quatre grands transporteurs (chiffre d'affaires supérieur à $18000 €$ et plus de 160 véhicules moteurs) dans ce secteur d'activité : Guirard Agediss, Guisnel Distribution, Vir Transport, Tem (réseau qui regroupe douze entreprises de transport indépendantes financièrement). Source : L'Officiel des transporteurs magazine, $\mathrm{N}^{\circ}$ spécial 1000 transporteurs du 26 novembre 2010, suppl. au n ${ }^{\circ} 2574$ de L'Officiel des transporteurs.
}

Les livraisons de l'entreprise représentent un volume de $5000 \mathrm{~m}^{3}$ par semaine, réparti sur une centaine de tournées en France et à l'export. Il faut noter que les retours ne sont pas toujours effectués à vide à la différence d'organisations de transport pour compte propre sur courte distance. L'entreprise utilise également le transport pour compte propre pour ses approvisionnements. Ce système de triangulation permet d'obtenir un taux de chargement au retour estimé à $70 \%{ }^{21}$, l'objectif étant d'atteindre les $80 \%$.

Les organisations de transport pour compte propre ayant une portée nationale, voire internationale s'expliquent d'une part, par des produits haut de gamme qui sont transportés avec des personnes délivrant un service complémentaire au produit. D'autre part, l'utilisation du transport pour compte propre sur longue distance par l'entreprise de meubles s'explique en raison du couplage des deux organisations de transport : les livraisons pour lesquelles le transport est plus fréquemment utilisé, mais également les approvisionnements. Les retours à vide doivent être minimisés afin de pérenniser l'organisation de transport.

Les organisations de transport pour compte propre restent difficiles à maintenir en raison de coûts importants qui croissent avec la distance avec notamment la prise en charge d'un découché du chauffeur. Les quelques cas qui ont recours au compte propre sur longue distance sont des entreprises qui réussissent à allier les livraisons aux clients - intérêt premier de leur activité - et les approvisionnements qui permettent de diminuer le coût de transport de la livraison.

\section{Conclusion}

Les organisations de transport pour compte propre sont en majorité des organisations de courte distance, toutefois elles sont plus complexes qu'il y paraît. En effet, cette courte distance ne résulte pas uniquement d'une externalisation des distances les plus longues ni d'une unique forme d'organisation spatiale des implantations. Ainsi, les organisations de type maillage territorial présentent une distance d'acheminement courte jusqu'au client, du fait d'un grand nombre de bases de départ et ce, que ce soit pour des raisons de contraintes liées au produit ou pour des raisons liées au service. Dans le cas d'une organisation régionale, les distances restent courtes mais sont supérieures à celles observées dans le cas d'un maillage territorial en raison d'une organisation centralisée autour d'un ou deux

${ }^{21}$ Entretien avec le directeur Transport et le logisticien Transport, le 23 juin 2008. 
pôles au sein d'une même région. Dans les deux cas, la livraison s'effectue dans une démarche de proximité avec le client et d'une meilleure connaissance de ses demandes leur permettant ainsi d'être réactifs mais également flexibles.

La principale différence entre ces deux types identifiés est la question de l'extension de ces organisations spatiales. Dans le cas du maillage, il existe, chez ces entreprises présentes dans l'ensemble de la France, une volonté de multiplier les implantations afin d'être le plus réactif tandis que dans le cas des organisations plus régionales, les entreprises cherchent à s'ancrer dans une unique région afin d'être les plus concurrentielles possibles, les clients situés hors de cette région sont livrés en compte d'autrui, car ils ne présentent pas un intérêt suffisant de par les volumes et les fréquences qu'ils génèrent.

Enfin, les organisations de transport pour compte propre à une échelle nationale voire internationale, bien que rares, sont possibles dans le cas de produits très spécifiques comme le montrent les deux exemples présentés. L'offre de transport est parfois inadaptée comme c'est le cas de l'entreprise de meubles qui livre jusqu'en Suède, faute d'identifier un professionnel de transport en adéquation avec ses besoins.

L'étude qualitative a permis d'illustrer la diversité des échelles d'utilisation du transport pour compte propre. Il en ressort que la qualité de service et la relation tissée avec le client semblent être les principaux arguments en faveur de ce type d'organisation de transport.

\section{Bibliographie}

1. Rodrigue J, Comtois C, Slack B (2009) The geography of transport systems. $2^{\text {nd }}$ Edition, Routeledge, New York, $352 \mathrm{p}$

2. Savy M (2006) Le transport de marchandises. Éditions d'Organisation, Eyrolles, Paris, $372 \mathrm{p}$.

3. Ministère de l'Écologie, du Développement Durable et de l'Énergie, Commissariat général au Développement durable (2006) Transport routier de marchandises, Disponible sur : http://www.statistiques.developpement-durable.gouv.fr/transports/ s/route.html [consulté le 03-09-2012]

4. Croucher P (1998) Insourcing. Logistics Focus 6(2): 3-6 et 8

5. Rodrigue J-P (2006) Challenging the Derived Transport Demand Thesis: Issues in Freight Distribution. Environment and Planning A 38(8):1449-1462.

6. Guilbault M, Soppé M (coord.) (2009) Apports des enquêtes chargeurs. Connaissance des chaînes de transport de marchandises et de leurs déterminants logistiques. Lavoisier, Coll. de I'INRETS, Actes n ${ }^{\circ} 120$, Bron, $220 \mathrm{p}$.

7. CEMT (Conférence Européenne des Ministres des Transports) (2001) Le transport routier de marchandises pour compte propre, Table ronde 115. OCDE, Paris, $140 \mathrm{p}$.

8. Patier D (2004) Transports de marchandises en ville : quelles spécificités quelles méthodes? La place du transport de marchandises en compte propre. DRAST, Laboratoire d'Economie des Transports, $61 \mathrm{p}$.

9. Cruz C (2010) How is own account transport well adapted to urban environments? Selected Proceedings of the $12^{\text {th }}$ World Conference on Transport Research, July 11-15, Lisbon - Portugal, pp B4-2883-18

10. Brunet R (1990) 6 : le maillage et le treillage. In: Brunet R, Dollfus O (eds.), Mondes nouveaux. Hachette, Reclus, Paris, pp 91-103

11. Baudelle G (1997) Quadrillage, treillage, maillage : discussion. L'Espace géographique 1: 79-80

12. Chanut O, Paché G, Wagenhausen F (2012) Logistique urbaine : refonder les logiques d'intermédiation. Revue Management et Avenir 51: 186-207 